\title{
Hepatic progenitor cell inhibition during embryonic period with high dose verapamil; liable joint to the cancer therapy
}

\author{
Uslu $\mathrm{S}^{1}$, Uysal A², Bilir A ${ }^{3}$, Soner BC ${ }^{4}$, Oktem $\mathrm{G}^{5}$ \\ Acibadem University Pathology Laboratory Technics, The School of Associated Health Sciences, Turkey. \\ gulperi.oktem@ege.edu.tr
}

\begin{abstract}
Cancer stem cells (CSCs) have been observed to share certain characteristics with normal stem cells. It was an important argument for cancer therapy and a successful progenitor inhibition could show us targeted cell type for a novel strategy. In this study, we aimed to constitute an inhibition in different stages of hepatic stem/progenitor cells (HPCs) with verapamil. Expression patterns of alpha-fetoprotein (AFP), c-kit (CD117) and p-glycoprotein were investigated in developing mouse on the embryonic day (E) 15, E18 and E21 to characterize early and late stages of HPCs. Proliferation inhibition with 5-Bromo-2-Deoxyuridin (BrdU) incorporation and maturation inhibition with PAS staining results were supported by morphometrical analysis during these periods. AFP, c-kit and p-glycoprotein immunoreactivity increased especially in E15 but decreased in E18 and E21 of the control groups during embryonic development. Verapamil treatment effected particularly E15 cells and immunoexpression of HPCs significantly decreased. Proliferation inhibition was observed in all embryonic days of mouse with verapamil and this drug inhibited not only maturation of HPCs in E18 and E21 embryos, but also decreased HPC number in the same embryonic period. According to our results, we estimated that similar to the early and late progenitor stages of HPCs, CSCc can also be in different stages in a heterogenic tumour bulk and the difficulty of CSC inhibition could be the main mechanism of tumour relapses. In this study, HPCs inhibition by verapamil in E15 was not observed in E18 and E21. As similar, CSCs treatments targeting different stages may be impotent to cells in tumour initiating cell stage. We can speculate that ineffectiveness of CSC-specific therapies may be attributed to the highly selective specificity of the treatment (Fig. 6, Ref. 28). Full Text in PDF www.elis.sk.

Key words: verapamil, cancer stem cells, p-glycoprotein, alpha-fetoprotein, embryonic period.
\end{abstract}

Hepatocellular carcinoma (HCC) is the fifth most common cancer worldwide and the third leading cause of cancer death (1). Re-growth of tumour and relapse of diseases have been thought to be driven by cancer stem cells (CSCs) (2). According to the CSC hypothesis, tumour growth is sustained by a subpopulation of cancer stem/progenitor-like cells and self-renewal and high clonogenic potential characteristics are shared by normal stem and CSCs $(3,4)$. This specialty brings new treatment modalities and targeted strategies. Embryonic period compromise various cell types in different embryonic days and developmental stages (5, 6). The early foetal liver contains hepatic progenitor cells (HPCs) since embryonic day (E) 14, which have the ability to differentiate either to hepatocytes or biliary epithelial cells. Liver HPCs can be isolated and cultured from the early embryonic liver and from

\footnotetext{
${ }^{1}$ Acibadem University Pathology Laboratory Technics, The School of Associated Health Sciences, Turkey, ${ }^{2}$ Ege University Faculty of Medicine, Department of Histology and Embryology Bornova, Izmir, Turkey, ${ }^{3}$ Istanbul University Istanbul Medical Faculty, Department of Histology and Embryology, Capa, Istanbul, Turkey, ${ }^{4}$ Selcuk University Faculty of Medicine Department of Pharmacology, Konya-Turkey, and ${ }^{5}$ Ege University Faculty of Medicine, Department of Histology and Embryology Bornova, Izmir, Turkey
}

Address for correspondence: G. Oktem, Ege University Faculty of Medicine, Department of Histology and Embryology, Izmir, Turkey. foetal liver (7). The hypothesis demonstrates that HPCs give rise to hepatocellular carcinoma cells since many tumours contain a mixture of mature cells and cells phenotypically resemble to HPCs. HPCs represents epithelial cell adhesion molecule (EpCAM) and alpha-fetoprotein (AFP) during embryonic period. Additionally, these molecules are highly expressed in hepatocellular carcinoma cells, CSCs and differentiation pathway is distinct from the process leading to reactivation of AFP expression in carcinoma cells (8).

Normal stem cells in embryonic period and CSC signalling resemble each other and they organize cancer modality. Embryonic period is a developmental permissive state and cells keep their stem cell progeny against potentially harmful molecules. In the course of time, molecules of embryonic development state lose their developmental potency and differentiate into foetal tissue cells. In the foetal stage, cells are usually unipotent and they give rise to specialized cell types. In this period of time, embryonic microenvironment shows tumour inhibiting properties $(9,10,11)$.

CSCs in hepatocellular carcinoma can be identified with several cell surface antigens including c-kit, CD133, CD90, CD44, OV6, and CD326 (EpCAM), or by selecting the side population (SP) cells by Hoechst dye-staining (12). CSCs with active drug efflux capability are multidrug resistant and pose a significant obstacle for the efficacy of chemotherapy. Recent studies suggested that high drug efflux cancer cells show stem-like cancer cell prop- 
erties, which are believed to be the cause for tumour initiation and recurrence. These cells are known to have highly efficient pumps for the Hoechst 33342 . Hoechst is able to enter living cells; it is also actively pumped out by ABC (ATP-Binding Cassette) transporters, which include p-glycoprotein and ABCG2 in human cells. Margaret Goodell's group was the first to identify a population of cells named SP cells. SP cells were all sensitive to verapamil. Verapamil is an inhibitor of $\mathrm{ABC}$ transporters and SP fractions are known to disappear during treatment. SP was blocked and totally ablated by verapamil (13). There is a great need for therapeutic reagents that are capable of eliminating these high drug efflux cancer stem cells and inhibitor effect of verapamil could be used in cancer treatment. In this study, we aimed to constitute inhibition in different stages of hepatic stem/progenitor cells with verapamil. A successful efflux inhibition could show us targeted cell type for therapy in HCC and could be a novel strategy for cancer therapy.

\section{Material and methods}

\section{Animal model and study design}

Wistar-Albino rats (n: 20) weighing 200-250 g were housed in 12:12 light/dark cycled rooms with the temperature at $22 \pm 3{ }^{\circ} \mathrm{C}$ and fed ad libitum. All experiments were approved by the Animal Ethics committee at the Ege University School of Medicine. Vaginal smears of rats were taken and stained with Giemsa stain every morning on 09:00 for five days. Female rats on their proestrous period were placed into a cage of one male rat overnight. The next morning, pregnancy of the female rats was determined by the presence of the sperm and vaginal plaque in the vaginal smear, which indicated gestational day $0.5(23,56,73)$. The lethal intraperitoneal (i.p.) rat dose of $60 \mathrm{mg} / \mathrm{kg} /$ day verapamil was administered (65). Verapamil was administered as a sublethal dose $(60 \mathrm{mg} / \mathrm{kg}$ ) on the 0 . day of pregnancy as $30 \mathrm{mg} / \mathrm{kg}$ i.p. with two doses. In this group (n: 5), the treatment caused miscarriage on the third day. Since a total dose of $60 \mathrm{mg} / \mathrm{kg} /$ day caused miscarriage, the dosage was changed as twice $10 \mathrm{mg} / \mathrm{kg}$ daily administered i.p. on the 0 .day of pregnancy. Although dosage has been decreased in this group (n: 6), miscarriages were observed on the 8, 9 and 10th day of pregnancy. As a consequence of miscarriages, the treatment protocol was rearranged as twice $10 \mathrm{mg} / \mathrm{kg}$ daily administered i.p verapamil on the 9th day of pregnancy where organogenesis begins. Verapamil treatment has been continued until the 21 st day of embryogenesis. Experimental groups constituted the Group A and B. Group A: E15 (15. embryonic day) (n: 11), E18 (n: 10) and E21 (n: 6) embryonic days non- drug application groups. Group B: E15 (n: 11), E18 (n: 10), and E21 (n: 8) embryonic day verapamil application groups. At the end of the study period, rats were sacrificed by withdrawal of blood by cardiac puncture under ketamine $(70 \mathrm{mg} / \mathrm{kg})$ and xylazine $(10 \mathrm{mg} / \mathrm{kg})$ anesthesia and liver tissue was dissected for analysis.

\section{Immunohistochemistry}

Paraformaldehyde fixation of liver tissues continued for 24 hours at $4{ }^{\circ} \mathrm{C}$ and processed for embedding in paraffin wax. Serial sections $5 \mu \mathrm{m}$ thick were cut using the microtome (Leica MR
2145, Heerbrugg, Switzerland). After dewaxing and rehydrating through a graded series of ethanol $(100 \%, 95 \%, 80 \%, 70 \%$, and $60 \%$ ) for 2 minutes in each concentration, sections were washed with distilled water and phosphate buffered saline (PBS) for 10 minutes and then treated with $2 \%$ trypsin (Sigma chemical Co., St. Louis, Missouri, USA) in $50 \mathrm{mM}$ Tris buffer (pH 7.5), at 37 ${ }^{\circ} \mathrm{C}$, for 15 minutes. Sections were delineated with a marker pen (Dako pen, Dako, Glostrup, Denmark.) and incubated in a solution of $3 \% \mathrm{H}_{2} \mathrm{O}_{2}$ for 15 minutes to inhibit endogenous peroxidase activity. To reduce non-specific background staining, slides were incubated at room temperature for 20 minutes in $0.3 \%$ bovine serum albumin/1xTris-buffered saline. Sections were incubated with primary antibodies; C-kit (1:50 dilution; Cell Marque), AFP (1:50 dilution, Dako) and p-glycoprotein (1:10 dilution, Neomarkers) for overnight at room temperature in a humid chamber. Then, sections were incubated with biotinylated secondary antibody (Histostain plus kit, Invitrogen Laboratories) and streptavidin conjugated to Horseradishperoxidase (Histostain plus kit, Invitrogen Laboratories) for 30 minutes in accordance with the manufacturer's instructions. Finally, sections were incubated with diaminobenzidine for 5 minutes to reveal immunolabeling. All dilutions and thorough washes between stages were performed using PBS. Sections were counterstained with Mayer's hematoxylin (Sigma chemical Co., St. Louis, Missouri, USA). After washing with tap water, sections were dehydrated through a graded ethanol series, cleared in xylene, and mounted with embedding agent (Entellan, Merck, Darmstadt, Germany). Negative control samples were processed in the same way except that the primary antibodies were omitted and replaced with PBS alone. All sections were analyzed under a light microscope (Olympus BX40, Tokyo, Japan). To determine the immunoreactivity of sections, a series of semi-quantitative analyses were performed in tissue materials. HSCORE was calculated using the following equation: $\mathrm{HSCORE}=\sum \mathrm{Pi}(\mathrm{i}+1)$, where is the intensity of labelling with a value of 1, 2 or 3, (weak, moderate, or strong, respectively) and $\mathrm{Pi}$ is the percentage of labelled cells for each intensity, varying from $0 \%$ to $100 \%$.

DNA synthesis (S-Phase) analyses via 5-Bromo-2-Deoxyuridin (BrdU) incorporation

BrdU (Neomarkers) injection has been adjusted to pregnant rats as $100 \mathrm{mg} / \mathrm{kg}$ intraperitoneally. One hour after injection embryos with E15, E18 and E21 has been dissected. The liver tissues, which have been dissected, were paraffin-embedded after routine tissue monitoring application. Sections were then incubated with $0.1 \%$ trypsin for antigen retrieval. After the final washing step, a nonspecific blocking reagent (Ultra-V-Block Lab Vision Co., Westinghouse, CA) was used to prevent nonspecific binding. Monoclonal mouse anti-BrdU antibody (Lab Vision Co, Westinghouse, CA, 1:50) was the primary antibody and secondary antibodies (1:50 Zymed Histostain ${ }^{\circledR}$ Puls Broad Spectrum) were used as biotinylated rabbit anti-mouse. After washing, peroxidaseconjugated streptavidin (Lab Vision Co., Westinghouse, CA) was applied and aminoethyl carbazole was used as chromogen. The sections were then counterstained with Mayer's Hematoxylin to augment the nuclear staining. For negative controls, adjacent 
sections were processed exactly with same steps excluding the primary antibodies.

\section{Periodic Acid-Schiff (PAS) staining}

After a routine preparation for histology, for the purpose to demonstrate glycogen synthase activity in hepatocyte, the samples were stained with $0.5 \%$ periodic acid solution then incubated with Schiff's reagents, washed with tap water, counterstained with hematoxylin solution, and thoroughly rinsed with tap water. Positive stain (purple red) was recorded under(Olympus BX40, Tokyo, Japan) microscope. Morphometrical analysis

Diameter measurements of Vena centralis

Liver tissues of $4 \mu \mathrm{m}$ thickness obtained from embryos of E15, E18 and E21 were stained with hematoxylin and eosin (H.E.) and reticulin for analysis of the morphology. Morphometrical analysis of reticular fibres was performed with Gordon and Sweets staining. Histopathology of liver section stained with hematoxylineosin stain. Formation of type III collagen in liver and basal membrane of portal vein and hepatic artery in hepatic triad was stained with reticulum. The images under microscopy (Olympus BX51, Tokyo) were obtained from 50 areas with $10 \mathrm{x}$ and transferred to a monitor through a device camcorder. Diameters of vena centralis were evaluated via UTHSCSA (The University of Texas Health Science Center At Santonio) Image Tool for Windows Version 1.28 image analyzing program.

Hepatic progenitor cell counting

The cytological features of the cell structure of liver were revealed with a greater detail from semi-thin sections from epoxy blocks and Toluidin blue staining was performed. All sections were analyzed under a light microscope (Olympus BX40, Tokyo, Japan) and 6 section areas were investigated. Cells were counted by using the Image-Pro Express image software (Media-Cybernetics). Measurements were performed by two independent researchers who were blind to the experimental groups. Sections were randomly chosen in all groups. The results were presented as the mean \pm SEM. The data were compared within the groups by using the result of analysis. Statistical differences were considered significant at $\mathrm{p}<0.05$. All statistical analyses were performed by using the Instat 3.05 software (GraphPad Software Inc., San Diego, CA).

\section{Results}

AFP and c-kit immunoreactivity changes during embryonic development

H-SCORE values of AFP and c-Kit (Fig. 1a) immunoreactivity in hepatic stem/progenitor cells during embryonic development were demonstrated. AFP immunoreactivity interestingly increased particularly in E15 (Fig. 2a) but decreased in E18 (Fig. 2c) and E21 (Fig. 2e) of the control groups during embryonic development. Nevertheless, AFP immunoreactivity effected verapamil treatment and statistics showed that a significant decrease was observed in the E15 liver control groups compared to E15 verapamil group $(134,91.50 \pm 0.86$ respectively $\mathrm{p}<0.05)$ (Fig. $2 b)$. There was no statistically significant difference in E18 and E21 control groups when compared the E18 and E21 verapamil group (78.50 \pm 2.98 , $76.25 \pm 3.70,74.75 \pm 4.02,70.50 \pm 3.30$ respectively $\mathrm{p}>0.05$ ) (Figs 2d, 2f). Similarly, c-kit immunoreactivity significantly decreased in E15 control group when compared to verapamil treatment group $(128.3 \pm 1.75,88.25 \pm 1.75$ respectively $\mathrm{p}<0.05)$ (Figs $2 \mathrm{~g}, 2 \mathrm{~h})$. On the other hand, there was no statistically significant difference in E18 and E21 control groups when compared the E18 and E21 verapamil group for c-kit immunoreactivity $(83.00 \pm 1.22,70.50 \pm 3.30$, $78.25 \pm 2.83,66$ respectively $\mathrm{p}>0.05$ ) (Figs $2 \mathrm{i}, 2 \mathrm{k}, 2 \mathrm{j}, 2 \mathrm{l})$.

Verapamil treatment effects proliferation of HPCs and p-glycoprotein immunoreactivity

According to the results of the Bromodeoxuridin immunoreactivity, Verapamil treatment significantly decreased the HPCSs proliferation index (Fig. 1b). Immunoreactivity of BrDu was strong in E15 control group (Fig. 3a) and decreased after Verapamil treatment (Fig. 3b) $(99.50 \pm 4.55,72.75 \pm 2.35$ respectively
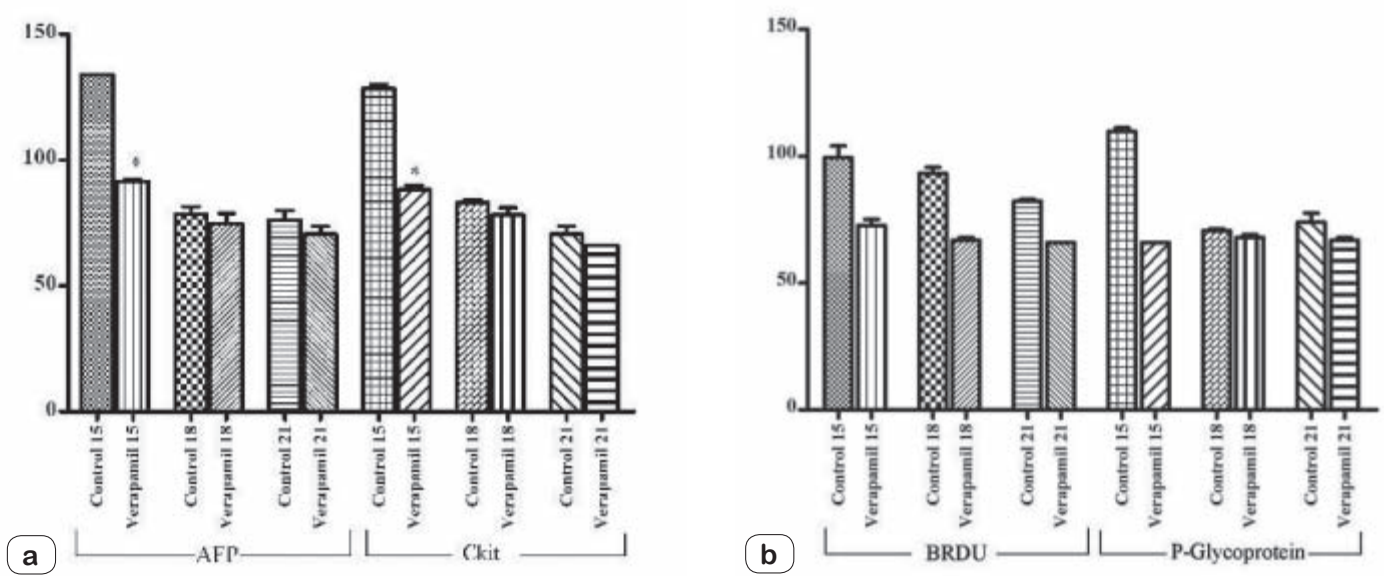

Fig. 1. H-SCORE values of AFP and c-Kit (a), BrdU and p-glycoprotein (b) immunoreactivity in hepatic stem/progenitor cells during embryonic development were demonstrated. 

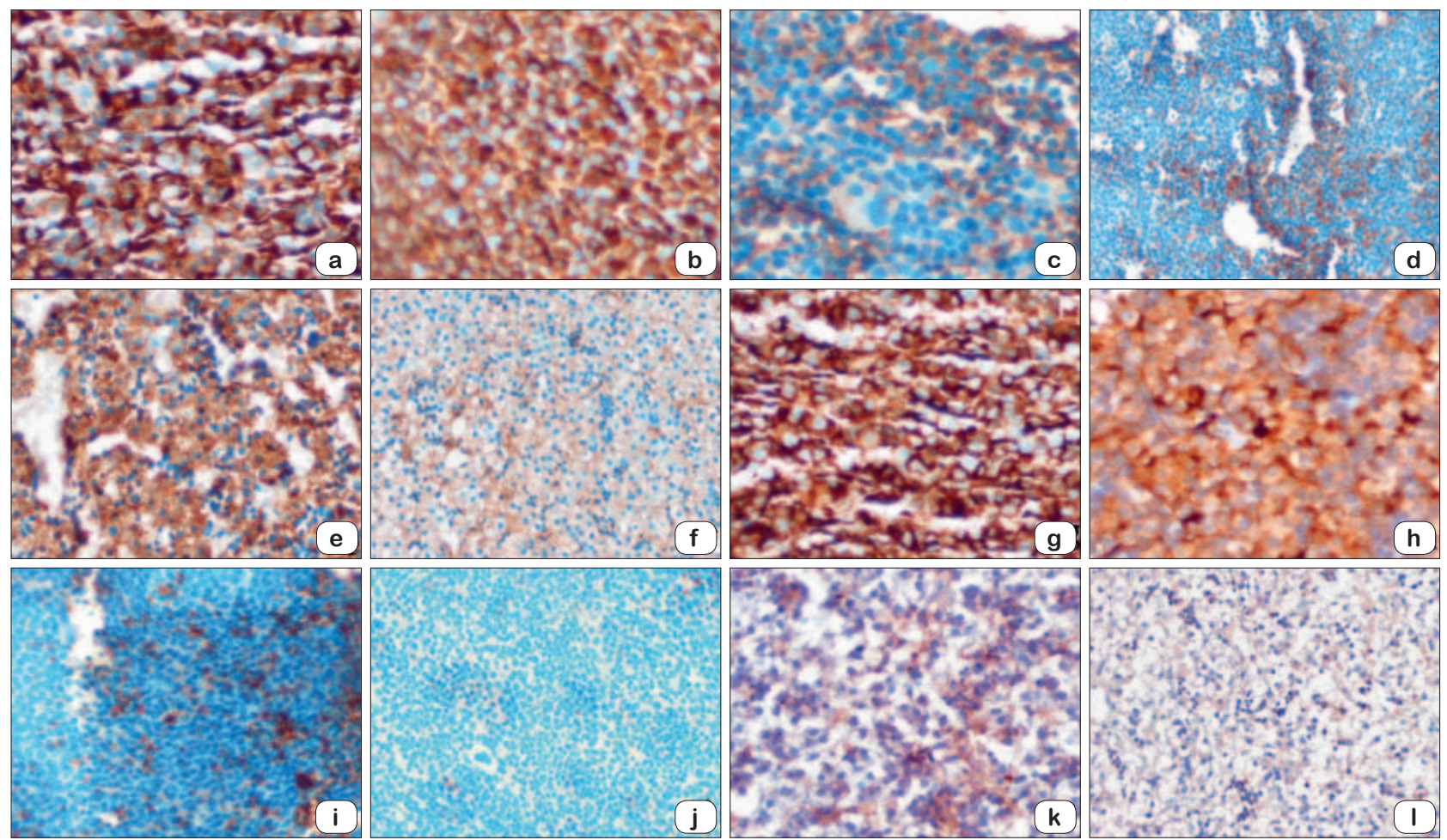

Fig. 2. Immunoreactivity displayed for AFP in E15 control (a) verapamil (b), E18 control (c) verapamil (d), E21 control (e) verapamil (f) and c-kit in E15 control (g) verapamil (h), E18 control (i) verapamil (k), E21 control (k) verapamil (l).
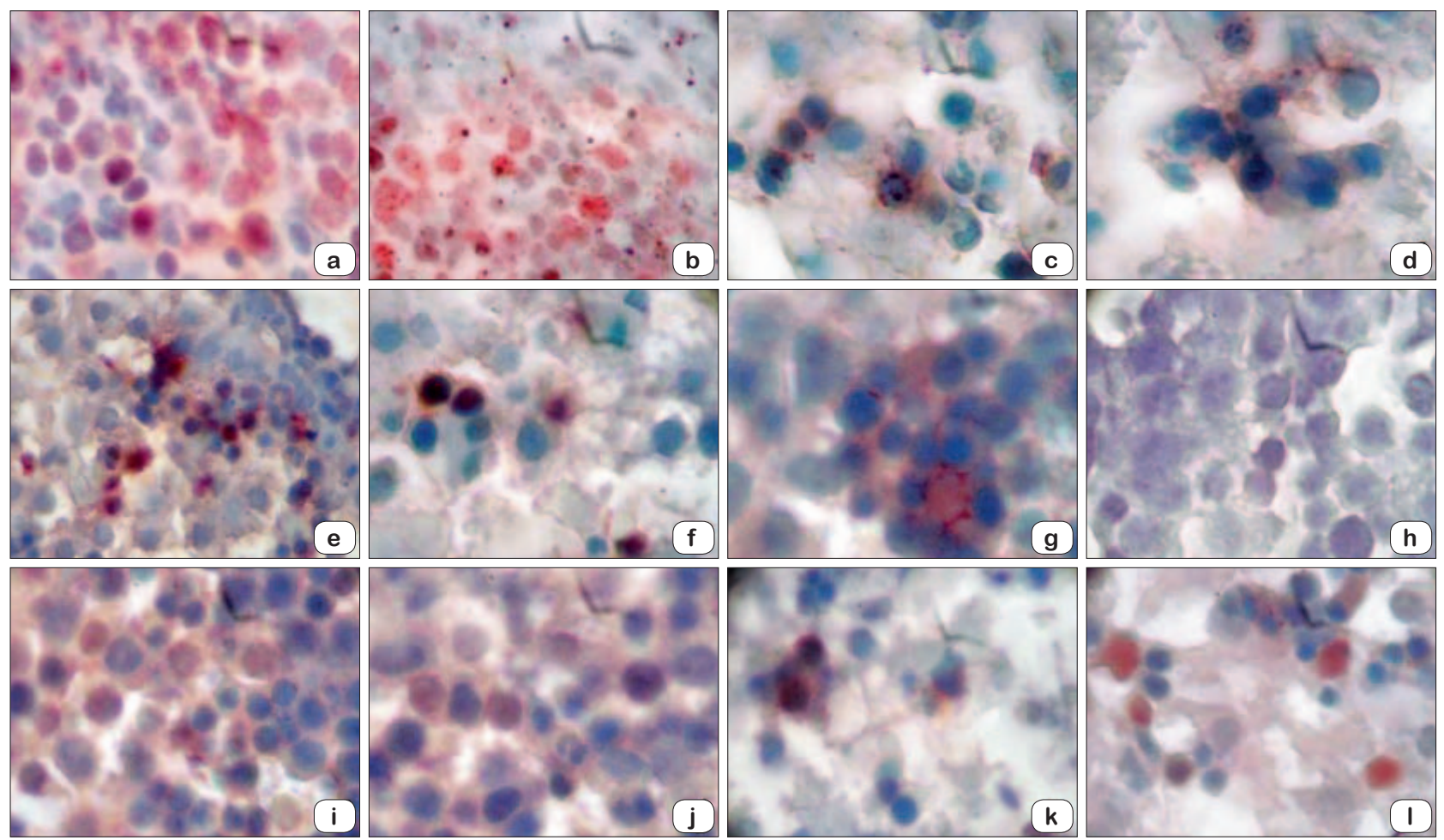

Fig. 3. BrdU immunoreactivity in E15 control (a) verapamil (b), E18 control (c) verapamil (d), E21 control (e) verapamil (f) and p-glycoprotein immunoreactivity in E15 control (g) verapamil (h), E18 control (i) verapamil (k), E21 control (k) verapamil (l) were demonstrated. 

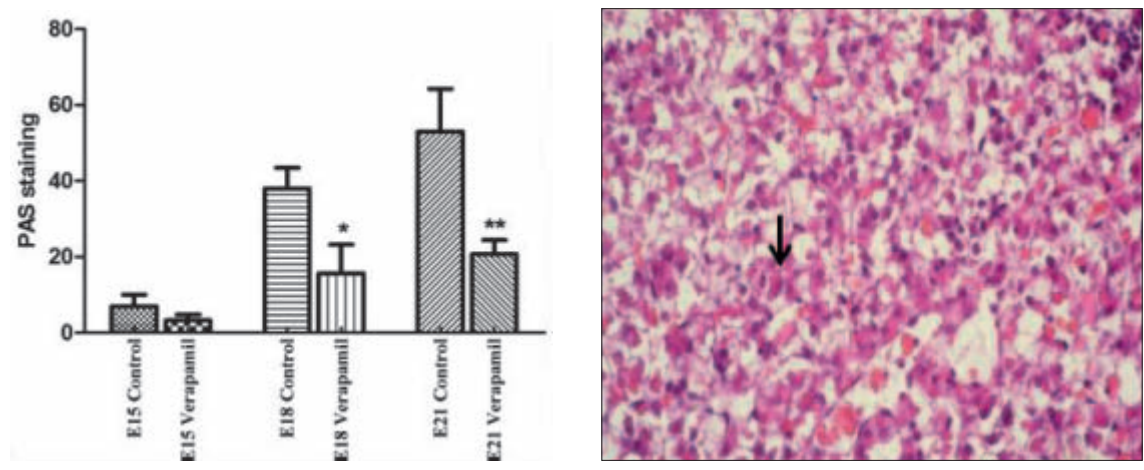

Fig. 4. PAS showed that there was not any staining in control and verapamil groups for E15. Nonetheless, strong PAS staining was observed in the control groups of E18 and E21 while marked decrease was observed in verapamil group of E18 and E21.
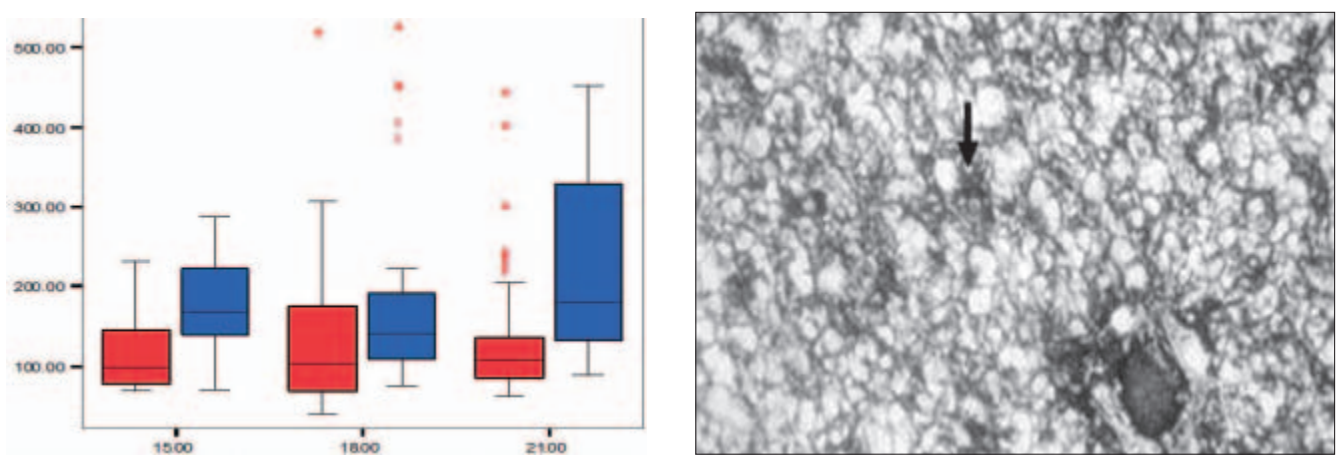

Fig. 5. Gordon and Sweets staining showed that there were not any differences in arrange of the reticular fibers. E15, E18 and E21 groups' measurements of vena centralis demonstrated that after verapamil treatment the diameter of these veins was increased significantly.
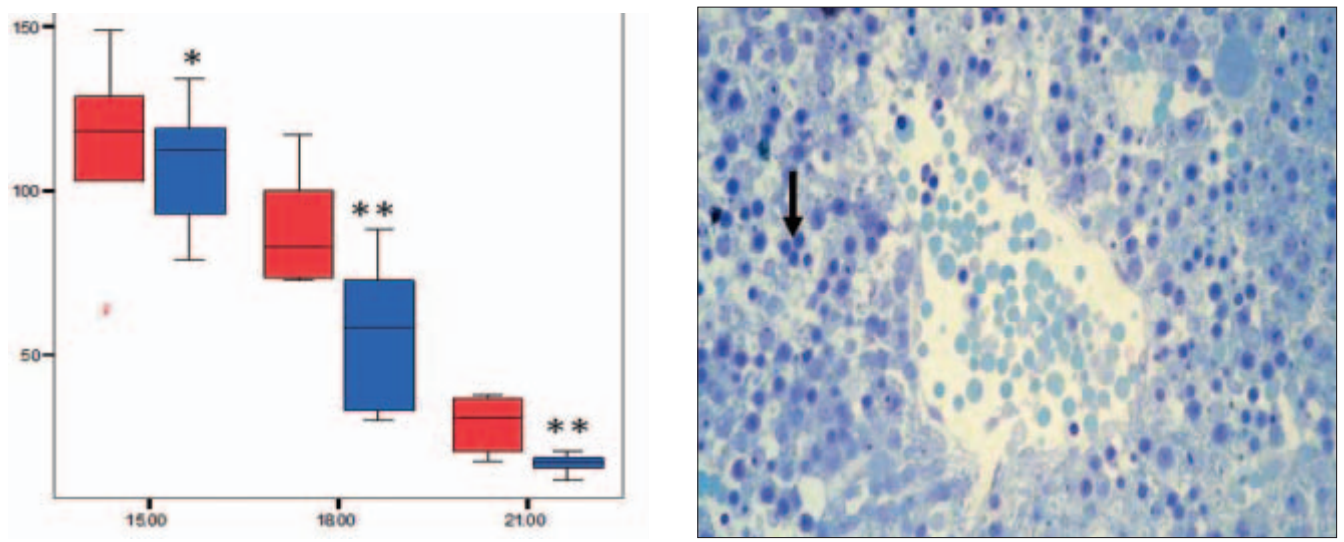

Fig. 6. Number of HPCs with toluidin blue showed that verapamil treatment significantly affected number of HPCs in the E18 and E21 verapamil when compared with their controls. However there were no statistically difference between E15 verapamil and E15 control groups.

p $<0.05$ ). BrdU immunoreactivity decreased E18 (Fig. 3c) and E21 (Fig. 3e) control groups according to E15 control group (Fig. 3a). Immunohistochemical analysis of rat liver cells revealed that immunoreactive cell number significantly decreased after Verapamil in both E18 (Fig. 3d) and ED 21 (Fig. 3f) according to the control groups $(67.00 \pm 1.00,66.00,93.25 \pm 2.3582 .25 \pm 0.75$ respectively $\mathrm{p}<0.05$ ). In this study, p-glycoprotein immunoreactivity showed a significant decrease in the E15 liver control groups (Fig. 3g) compared to the E15 verapamil group (Fig. 3h) $(109.8 \pm 1.43,66.01$ respectively $\mathrm{p}<0.05)$. There was no statistically significant difference in E18 (Fig. 3i) and E21 (Fig. 3k) control groups when compared the E18 (Fig. 3k) and E21 (Fig. 31) verapamil group (70.75 \pm 0.75 , $74.00 \pm 3.55,68.00 \pm 1.15,67.00 \pm 1.00$ respectively $\mathrm{p}>0.05)$.

\section{Verapamil is impressive on maturation of HPCs}

PAS staining method were used to identify glycogen in tissues and mature cells can also be positively stained. We tested the effect of verapamil in different stages of liver cell and demonstrated that verapamil significantly inhibited the terminal differentiation of HPCs. In this study, there were no PAS staining 
in the control and verapamil groups for E15. Nonetheless, strong PAS staining was observed in the control groups of E18 and E21 while a marked decrease was observed in the verapamil group of E18 and E21 (Fig. 4).

\section{Diameter of the vena centralis and number of HPCs after vera- pamil treatment}

The results of this experiment showed that there were no differences in the arrangement of the reticular fibres and the morphometrical analysis of the sections among all experimental groups. On the other hand, in E15, E18 and E21 groups measurements of vena centralis demonstrated that after verapamil treatment, the diameter of these veins were significantly increased in $(p<0.005)$ (Fig. 5). The number of HPCs with toluidin blue showed that verapamil treatment significantly affected the number of HPCs in the E18 and E21 verapamil when compared to their controls. However, there were no statistically difference between E15 verapamil and E15 control groups (Fig. 6).

\section{Discussion}

During embryonic development, different stages of HPCs exist in liver. These cells constitute hepatocellular carcinoma - cellular composition in the postpartum period of life $(14,17)$. In our study, foetal liver cells with various embryonic periods were investigated to identify the effective inhibition period of differentiating HPCs and determination of the targeted cell types for assistance of cellular therapies. With this aim, our results showed that the inhibition of HPCs is possible during E15 with verapamil treatment when cells situated in early stage. However, in due course, cells passed to the late stage and it was not possible to inhibit these HPCs with verapamil. Proliferation inhibition showed all embryonic days of mouse with verapamil and verapamil inhibited not only maturation of HPCs in E18 and E21 embryos, but also decreased HPC number in the same embryonic period. C-kit, AFP and p-glycoprotein immunoreactivity were significantly decreased in E15 HPCs when compared to E18 and E21. AFP is a typical marker of hepatoblast and foetal hepatocytes. This molecule enables to pursue differentiation, retro-differentiation, ontogeny, oncodevelopmental situation and postnatal repression of cells for the commitment of the liver lineages (18). AFP positive cells in hepatocellular carcinoma had features of hepatic stem/progenitor cells and correlated with prognosis and invasiveness of tumour (12).

Konishi et al. demonstrated that HPCs derived from foetal liver tissue until E13.5 could be candidates for hepatic stem cell in the foetal liver (19). After this period, HPCs possibly maintain differentiation and liver progenitor cell marker AFP significantly decreases. AFP is in close contact with vimentin and during E11.5 and E13.5 almost all the vimentin-positive cells are AFP-positive, yet the percentage of AFP-positivity in vimentin expressing cells decreased dramatically at E17.5. On the other hand, until E17.5 vimentin- positive cells were BrdU- positive (20). Our study supported this data and we indicated a significant decrease in AFP at E18 and E21. It is worthy noticing that verapamil inhibited HPCs markers and proliferation in the early period of hepatic stem cell.
Calcium is almost universal intracellular messenger controlling a diverse range of cellular processes such as gene transcription, muscle contraction and cell proliferation (21). Extracellular calcium has an important role in cell-cell adhesion in mammalian embryos' preimplantation process (20). Intracellular free ionized calcium has a key role in fertilization, oocyte maturation/activation and cortical granule exocytosis $(23,25)$. Previous studies have shown that mice embryos treated with calcium channel blockers had growth retardation (25). Our preliminary study with $60 \mathrm{mg} /$ $\mathrm{kg}$ /day dose of verapamil has shown that besides its inhibitory effect on HPCs, $60 \mathrm{mg} / \mathrm{kg}$ /day dose may also cause miscarriages via inhibiting implantation.

PAS staining is primarily used to identify glycogen in tissues although glycoprotein and proteoglycans and glycogen stores are characteristic features of mature hepatocytes (26). In this study, verapamil treatment inhibited not only maturation of HPCs in E18 and E21 embryos, but also decreased HPC number in the same embryonic period. Since it is possible to assume that E15 mouse foetal liver early progenitor cells are affected from the inhibitory effect of verapamil and differentiation is not continues in this period. The effect of inhibition does not occur following this period in late progenitory cells. Human hepatic stem cells most likely give a rise to hepatocellular carcinoma and hepatic cholangiocarcinoma and quite likely, hepatocellular carcinoma arises from HPCs (27). Tumour cells contain a mixture of different stage of cells between early progenitors and mature hepatocytes (28). The main result of this study was that the inhibition of HPCs in late stage was possible with verapamil. However, in this study we had also examined the other different effects of verapamil on late stage mouse embryo. Realization of CSCs in tumours has changed our view of carcinogenesis and chemotherapy. Inhibition of CSCs remains a challenge for drug development and treatment strategies. Tumour mass is composed of heterogenous cells and CSCs in tumour mass can be in various differentiation stages. Similar to the early and late progenitor stages of HPCs, CSCc can also be in different stages in a heterogenous tumour bulk and the difficulty of CSC inhibition could be the main mechanism of tumour relapses. Some CSCs that has been marked with appropriate surface markers might be inhibited in-vivo and/or in-vitro by various agents but the early stage CSCs in tumour bulks might be the reason for tumour progression and relapses. In this study, HPCs inhibition by verapamil in E15 was not observed in E18 and E21. As similar, CSCs treatments targeting different stages may be impotent to cells in tumour initiating cell stage. We can speculate that ineffectiveness of CSC-specific therapies may be attributed to the highly selective specificity of the treatment.

\section{References}

1. El-Serag HB. Epidemiology of hepatocellular carcinoma. Clin Liver Dis 2001; 5: 87-107.

2. Pardal R, Clarke MF, Morrison SJ. Applying the principles of stemcell biology to cancer. Nat Rev Cancer 2003; 3: 895-902.

3. Vermeulen L, Sprick MR, Kemper K, Stassi G, Medema JP. Cancer stem cells-old concepts, new insights. Cell Death Differ 2008; 15: 947-958. 
4. Ponti D, Costa A, Zaffaroni N, Pratesi G, Petrangolini G et al. Isolation and in vitro propagation of tumorigenic breast cancer cells with stem/ progenitor cell properties. Cancer Res 2005; 65: 5506-5511.

5. Pappa KI, Anagnou NP. Novel sources of fetal stem cells: where do they fit on the developmental continuum? Regen Med 2009; 4 (3): 423-433.

6. Rogler LE. Selective bipotential differentiation of mouse embryonic hepatoblasts in vitro. Am J Pathol 1997; 150: 591-602.

7. Petkov PM, Zavadil J, Goetz D, Chu T, Carver R, Rogler CE, Bottinger EP, Shafritz DA, Dabeva MD. Gene expression pattern in hepatic stem/progenitor cells during rat fetal development using complementary DNA microarrays. Hepatology 2004; 39 (3): 617-627.

8. Cantz T, Zuckerman DM, Burda MR, Dandri M, Göricke B, Thalhammer S, Heckl WM, Manns MP, Petersen J, Ott M. Quantitative gene expression analysis reveals transition of fetal liver progenitor cells to mature hepatocytes after transplantation in uPA/RAG-2 mice. Am J Pathol 2003; 162 (1): 37-45.

9. Strizzi L, Hardy KM, Seftor EA, Costa FF, Kirschmann DA, Seftor RE, Postovit LM, Hendrix MJ. Development and cancer: at the crossroads of Nodal and Notch signaling. Cancer Res 2009; 69 (18): 7131-7134.

10. Kho AT, Zhao Q, Cai Z, Butte AJ, Kim JY, Pomeroy SL, Rowitch DH, Kohane IS: Conserved mechanisms across development and tumorigenesis revealed by a mouse development perspective of human cancers. Genes Dev 2004; 18 (6): 629-640.

11. Lepourcelet M, Tou L, Cai L, Sawada J, Lazar AJ, Glickman JN, Williamson JA, Everett AD, Redston M, Fox EA, Nakatani Y, Shivdasani RA. Insights into developmental mechanisms and cancers in the mammalian intestine derived from serial analysis of gene expression and study of the hepatoma-derived growth factor (HDGF). Development 2005; 132 (2): 415-427.

12. Oishi N, Wang XW. Novel therapeutic strategies for targeting liver cancer stem cells. Int J Biol Sci 2011; 7 (5): 517-535.

13. Goodell MA, McKinney-Freeman S, Camargo FD. Isolation and characterization of side population cells. Methods Mol Biol 2005; 290: 343-352.

14. Michalopoulos GK, DeFrances MC. Liver regeneration. Science 1997; 276: 60-66.

15. Shafritz DA, Oertel M, Menthena A et al. Liver stem cells and prospects for liver reconstitution by transplanted cells. Hepatology 2006; 43 (Suppl 1): 89-98.
16. Theise ND, Yao JL, Harada K et al. Hepatic 'stem cell' malig-nancies in adults: four cases. Histopathology 2003; 43: 263-271.

17. Yao Z, Mishra L. Cancer stem cells and hepatocellular carci-noma. Cancer Biol Ther 2009; 8: 1691-1698.

18. Kuhlmann WD, Peschke P. Hepatic progenitor cells, stem cells, andAFP expression in models of liver injury. Int J Exp Pathol 2006; 87 (5): 343-359.

19. Konishi S, Yasuchika K, Ishii T, Fukumitsu K, Kamo N, Fujita N, Ikai I, Uemoto S. A transmembrane glycoprotein, gp38, is a novel marker for immature hepatic progenitor cells in fetal mouse livers. In Vitro Cell Dev Biol Anim 2010; 47 (1): 45-53.

20. Sugano N, Wakino S, Kanda T, Tatematsu S, Homma K, Yoshioka $\mathbf{K}$ et al. T-type calcium channel blockade as a therapeutic strategy against renal injury in rats with subtotal nephrectomy. Kidney Int 2008; 73 (7): 826-834.

21. Bootman MD, Collins TJ, Peppiatt CM, Prothero LS, MacKenzie L, De Smet P, Travers M, Tovey SC, Seo JT, Berridge MJ, Ciccolini F, Lipp P. Calcium signalling--an overview. Semin Cell Dev Biol 2001; 12 (1): 3-10.

22. Pey R, Vial C, Schatten G, Hafner M. Increase of intracellular Ca2+ and relocation of E-cadherin during experimental decompaction of mouse embryos. Proc Natl Acad Sci USA 1998; 95 (22): 12977-12982.

23. Whitaker M. Calcium signalling in early embryos. Philos Trans R Soc Lond B Biol Sci 2008; 12; 363 (1495): 1401-1418.

24. Whitaker M. Calcium at fertilization and in early development. Physiol Rev 2006; 86 (1): 25-88.

25. Stein G, Srivastava MK, Merker HJ, Neubert D. Effects of calcium channel blockers on the development of early rat postimplantation embryos in culture. Arch Toxicol 1990; 64 (8): 623-638.

26. Bi Y, Huang J, He Y, Zhu GH, Su Y, He BC et al. Wnt antagonist SFRP3 inhibits the differentiation of mouse hepatic progenitor cells. J Cell Biochem 2009; 108 (1): 295-303.

27. Tee LB, Kirilak Y, Huang WH, Smith PG, Morgan RH, Yeoh GC. Dual phenotypic expression of hepatocytes and bile ductular markers in developing and preneoplastic rat liver. Carcinogenesis 1996; 17 (2): 251-259.

28. Mansuroglu T, Baumhoer D, Dudas J, Haller F, Cameron S, Lorf T, Füzesi L, Ramadori G. Expression of stem cell factor receptor c-kit in human nontumoral and tumoral hepatic cells. Eur J Gastroenterol Hepatol 2009; 21 (10): 1206-1211. 